\title{
COMMENTARY ON TAGUCHI'S PARAMETER DESIGN WITH DYNAMIC CHARACTERISTICS
}

\author{
ARDEN MILLER \\ Department of Statistics, University of Auckland, Private Bag, Auckland, New Zealand \\ AND \\ C.F.J. WU \\ Department of Statistics, University of Michigan, Ann Arbor, MI 48109-1027, U.S.A.
}

A major recent advance in quality engineering and industrial statistics is the development and applications of robust parameter design for product and process improvement. This methodology, pioneered by the Japanese quality expert Dr. Genichi Taguchi, has now been widely used in industries in many countries, and many industrial case studies can be found in proceedings of statistical and quality meetings and in publications by private consulting organizations such as the American Supplier Institute. The industrial statistics research community has given a lot of attention to parameter design, which is evidenced by the rapid increase of publications on the subject. A comprehensive discussion on parameter design can be found in a Technometrics article edited by Nair. ${ }^{1}$ The main purpose of this commentary is to point out some problems associated with Taguchi's approach to parameter design for 'dynamic' characteristics and to outline sound alternatives. Owing to the abbreviated nature of this commentary, readers may wish to refer to References 2 and 3 for technical details.

Taguchi ${ }^{4}$ defined two general classes of application for his parameter design methodology which he referred to as 'static characteristics' and 'dynamic characteristics'. Most of the interest in parameter design generated in the statistical literature has focused on static applications. ${ }^{1,5}$ With a few exceptions such as References 6 and 7, relatively little work has appeared on the dynamic problem. Static characteristic applications involve situations where the goal can be summarized as getting the value of a quality characteristic of interest, $Y$, as close as possible to a single specified target value. For example, Kacker and Shoemaker ${ }^{8}$ consider a process which deposited layers of silicon on top of silicon wafers. The goal was to make the thickness of the deposited layer as close as possible to a target value. Dynamic characteristic applications, on the other hand, involve situations where the performance of the system is determined by the relationship between a signal factor, $M$, and an observed response, $Y$. In these cases, the response is required to assume different values as a result of changes in the signal factor. Since the term 'dynamic' is somewhat misleading, Miller and $\mathrm{Wu}^{3}$ suggested that such applications be referred to as signal-response systems. Here we will use the two terms interchangeably.

One general class of signal-response systems is multiple target applications. For example, Yano (Reference 9, p. 293) describes a process where parts are machined using a lathe. Different applications required machined parts with different degrees of surface roughness to be produced. As it was known that the feed rate of the tool bit could be used to alter surface roughness, feed rate was selected as the signal factor. How reliably surface roughness can be controlled by adjusting feed rate depends on the characteristics of the relationship between surface roughness and feed rate. It was thought that other factors such as lathe, cutting speed, depth of tool cut, type of tool cut, corner radius, cutting edge angle, front escape angle, and side scoop angle may affect this relationship, so an experiment was conducted to find preferred settings for these factors. A second class of signal-response applications is measurement systems. A measurement system is the process used to obtain an estimate of some quantity of interest for a given unit or sample, and may include the sampling procedure, sample preparation, and calibration, as well as the actual measurement process. The true amount of the quantity present can be considered as an input signal, $M$, which the system converts into a measured value or response, $Y$. The precision with which $M$ can be estimated based on $Y$ is determined by the characteristics of the relationship between $M$ and $Y$.

Taguchi's experimentation strategy for dynamic applications is a modification of his approach for static applications. Experimental factors are divided into three groups: control factors, noise factors, and the signal factor. Control factors represent aspects of the process which can easily be adjusted by the operator. These represent the opportunities available to modify the process in order to improve performance. Noise factors represent conditions which are 
thought to affect the process but are too difficult or expensive to control during the usual operation of the system. These factors cause variation in the process and thus have an adverse effect on performance. Performance will be improved if the process can be made insensitive to noise factors. Taguchi advocates a product array design with the control factor levels determined by an inner array and the signal and noise factor levels determined by an outer array. For each combination of control factor levels in the inner array, observations are made corresponding to each run in the outer array and a fitted linear model for the response as a function of the signal factor, either $Y=\beta_{1} M$ or $Y=\beta_{0}+\beta_{1} M$ depending on the circumstances, is obtained using least squares. The dynamic signal-to-noise (SN) ratio for the ith control factor combination is defined as

$$
\mathrm{SN}_{i}=\frac{\hat{\beta}_{1 i}^{2}}{\mathrm{~S}_{i}^{2}}
$$

where $\hat{\beta}_{\|}$is the least squares estimate for $\beta_{1}$, and $s_{i}^{2}$ is the standard estimate of residual variation.

Taguchi assumes that the ideal signal-response relationship is a deterministic linear function of the form $Y=\beta_{0}^{\prime}+\beta_{1}^{t} M$ or $Y=\beta_{1}^{\prime} M$ where $\beta_{0}^{\prime}$ and $\beta_{1}^{t}$ represent the ideal values of the intercept and slope respectively, and ' $t$ ' denotes target (not a power of $\beta)$. Any departures from the ideal function indicates the system is not working efficiently and results in a loss of quality. A two-step optimization procedure is used to optimize the true signal-response relationship. First the calculated SN ratios or more commonly the logarithms of these ratios are treated as the response over the control array runs. Standard analyses such as analysis of variance or normal plots of estimated effects are used to determine control factor settings which maximize the dynamic SN ratio. Then 'adjustment factors' are used to move $\beta_{0}$ and $\beta_{1}$ to the target values. These adjustment factors are factors which have been identified, either from previous knowledge or from the results of the experiment, as having no effect on the dynamic $S N$ ratio but which are able to adjust the values of $\beta_{0}$ and $\beta_{1}$.

Taguchi's methodology can be motivated using a quadratic loss model. Suppose we assume that the ideal signal-response function is linear, and that at a fixed value of $M$, loss is proportional to the squared deviation of $Y$ from its ideal value as defined by the ideal function. In equation (1), $s_{l}^{2}$ is proportional to the sum of the squared residuals and so could be interpreted as being a measure of loss over the outer array runs provided that the fitted signal-response function is the same as the ideal function. Now if it is assumed the signal-response function can be modified in such a manner that any change in $\beta_{1}^{2}$ is accompanied by a corresponding change in the squared deviations then (1) would be a measure of (loss) $)^{-1}$ given that the signal-response function is adjusted to target.

Taguchi's dynamic SN ratio approach has several deficiencies. First, the assumption that the ideal signal-response relationship is linear is unnecessarily restrictive. There are examples, such as electronic amplification circuits, where a linear relationship is required, and Liggett ${ }^{10}$ specifically addresses such systems. However, in many other applications linearity does not hold. Certainly, both measurement systems and multiple target systems can operate very successfully based on a non-linear signal-response relationship provided the form of the relationship is well understood. In addition, the dynamic SN ratio lumps all the information about the performance of the system into a single measure for each combination of control factors. This measure is affected by the sensitivity of the response to the signal factor. departures of the signal-response relationship from linearity, variation due to each of the noise factors, and variation due to other uncontrolled sources. As a result useful information about how specific control factors affect these different aspects of the system is lost. Such information may aid the experimenters in understanding the system and point them in promising directions for system improvement. Also, the assumption that adjustment factors which do not affect the $\mathrm{SN}$ ratio are either known to exist or can be reliably identified is unwarranted in many situations. Finally, the product array designs advocated by Taguchi are often unnecessarily large, which results in inefficient experiments. See Reference 11 for a similar point in the context of static applications.

An alternative strategy for signal-response application is to model the response as a function of all the experimental factors (control, noise, and signal), which is an adaptation of the response modelling approach suggested by Welch et al. ${ }^{12}$ and Shoemaker, Tsui and $\mathrm{Wu}^{11}$ for static applications. There are some clear advantages to this approach over Taguchi's methodology. First, all experimental factors can be varied according to a single design array which allows much greater flexibility in selecting a design. This will often result in a more economical experiment than can be obtained using a product array design. Secondly, the response model will provide a detailed picture of how control factors interact with the noise factors and the signal factor. This insight will often suggest promising avenues for future research. Grove and Davis ${ }^{7}$ provide an interesting discussion of the response modelling approach applied to a signal-response application.

In general, we recommend a modification to the response model approach which we refer to as response function modelling. The idea is to model the signal-response relationship as a function of the control and noise factors which often simplifies the analysis process. A product array design is used but for this approach the control and noise factors are put in the inner array and only the signal factor is 
in the outer array. Note that this type of design is particularly suited to a split-plot experiment with control and noise factors assigned to main units and the signal factor assigned to split units. For many signal-response applications this will be an attractive option since the signal factor is often easy to adjust relative to control and noise factors. For each inner array run, a fitted parametric model of the signal-response relationship is obtained using the observations corresponding to the signal levels in the outer array. The fitted parameters of these models are then analysed as functions of the control and noise factors. For example, if a linear model of the form $Y=\beta_{0}+\beta_{1} M+\epsilon$ where $\epsilon \sim \mathrm{N}\left(0, \sigma^{2}\right)$ is a suitable approximation of the signal-response function, then estimates of $\beta_{0}, \beta_{1}$ and $\sigma^{2}$ are obtained for each run in the inner array. These estimates are then analysed as functions of the control and noise factors. In this manner, a clear picture of how the control and noise factors affect the signal-response relation is obtained. Usually, it will be possible to identify preferred settings of the control factors directly from this analysis by considering the function of the system being studied. As an example, a multiple target system will have a required set (or range) of target values and a usable range of the signal factor. The control factor settings must allow all required target values to be obtained for levels of the signal factor in the usable range. Further, the sensitivity of the signal-response relationship to each of the noise factors should be made as small as possible and the size of residual variation should be minimized. Finally, provided that all targets remain obtainable, it may be advantageous to make the sensitivity of the response to the signal as small as possible since any error in setting the signal factor will be transmitted to the response through the gradient of the signal-response relationship. Therefore, selection of control factor settings is made on the basis of optimizing these different aspects of the signal-response relationship. In most cases this is straightforward.

A problem can arise if some control factors have conflicting effects on different aspects of the signalresponse relationship. For example, setting a particular control factor to minimize the variation due to one of the noise factors may conceivably increase the variation due to a second noise factor. In such cases, a formal performance measure can be devised which combines the different aspects of a desirable signal-response relationship into a single measure. Preferred control factor settings can be identified by evaluating this measure for different control factor combinations using the fitted model for the signalresponse relationship. Miller and $\mathrm{Wu}^{3}$ provides a discussion of suitable performance measures for signal-response systems as well as illustrating the response function modelling procedure.

Signal-response or dynamic characteristics are an important application of robust parameter design which has unfortunately been somewhat neglected in the statistical literature. We recommend the response function modelling approach since it retains the advantages of the more general response modelling approach while often simplifying the analysis and interpretation of the experiment. A diverse range of models can be entertained for the signal-response relationship without making the modelling procedure too complicated. The following classes of models will often prove useful:

1. Linear models in $M$ (possible after a suitable transformation of $M$ and/or $Y$ )

2. Polynomial functions of $M$

3. Non-linear models suggested by engineering knowledge

In all these cases, the response function modelling procedure simply consists of fitting the chosen signal-response relationship for each run in the inner array and then modelling the fitted parameters as functions of the control and noise factors. In this way the effects of control and noise factors on different aspects of the signal-response relationship are evaluated directly. (For example if a linear model is used for the signal-response function, then it is readily apparent from the analysis which control and noise factors affect the slope and which ones affect the intercept.) Usually settings of the control factors can be selected directly from the fitted signal-response model.

\section{REFERENCES}

1. V. N. Nair (ed), 'Taguchi's parameter design: a panel discussion', Technometrics, 34, 127-161 (1992).

2. A. E. Miller and C. F. J. Wu, 'Improving a calibration system through designed experiments', Research Report 9106, Institute for Improvement in Quality and Productivity, University of Waterloo, 1991.

3. A. E. Miller and C. F, J. Wu, 'Parameter design for signalresponse systems', University of Michigan Technical Report No. 251. Revised for Statistical Science, 1996.

4. G. Taguchi, System of Experimental Design, Unipub/Kraus International Publications, White Plains, New York, 1987.

5. G. E. P. Box, 'Signal-to-noise ratios, performance criteria, and transformations', Technometrics, 30, 1-40 (1988).

6. M. S. Phadke, Quality Engineering Using Robust Design, Prentice Hall, Englewood Cliffs, NJ, 1989.

7. D. M. Grove and T. P. Davis, Engineering, Quality \& Experimental Design, Longman Scientific \& Technical, England, 1992.

8. R. N. Kacker and A. C. Shoemaker, 'Robust design: a costeffective method for improving manufacturing processes', AT\&T Technical Joumal, 65, 39-50 (1986).

9. H. Yano, Metrological Control: Industrial Measurement Management, Asian Productivity Organization, Tokyo, 1991.

10. W. Liggett, 'Project planning for sequential parameter-design experiments', Technical Repor, Statistical Engineering Division, NIST, Gaithersberg, MD, 1991.

11. A. C. Shoemaker, K. L. Tsui and C. F. J. Wu, 'Economical experimentation methods for robust design', Technometrics, 33. 415-427 (1991).

12. W. J. Welch, T. K. Yu, S. M. Kang and J. Sacks, Computer experiments for quality control by parameter design', Journal of Quality Technology, 22, 15-22 (1990)

Authors' biographies:

Arden Miller is a Lecturer at the University of Auckland. He received his Ph.D. in Statistics from the University of 
Waterloo in 1993. He is a member of the Statistical Society of Canada, the American Statistical Association, and the New Zealand Statistical Association. Arden's areas of interest include robust parameter design, design of experiments, and quality improvement.

C. F. Jeff Wu is Professor and Chair, Department of Statistics and also Professor of Industrial and Operations Engineering at the University of Michigan, Ann Arbor. He was formerly the GM/NSERC Chair in Quality and
Productivity at the University of Waterloo. He is a Fellow of the Institute of Mathematical Statistics, and of the American Statistics Association, co-winner of the 1990 Wilcoxon Prize for the best practical application paper in Technometrics, and of the 1992 Brumbaugh Award for the single most important paper to quality control among the publications sponsored by the American Society for Quality Control. He also received the prestigious COPSS (Committee of Presidents of Statistical Societies) Award in 1987. 